\title{
Optimizing Offline and Online Selling Prices in a Monopolistic Environment
}

\author{
Hongkai Zhang \\ East Central University
}

\author{
Jiehui Ma \\ University of Wisconsin-Whitewater
}

James Rauch

East Central University

Based on two linear deterministic demand models, the sales revenue function of a multivariate quadratic form is formulated in this paper to determine the optimal offline and online selling prices for a retailer in a monopolistic environment. The impacts of the uniform delivery lead time on the retailer's optimal pricing scheme and profitability are analytically examined. A numerical study is conducted to demonstrate these impacts.

\section{INTRODUCTION}

A retailer may sell its products via multiple retail channels. For example, major retailers such as Sears, J. C. Penney, and Walmart currently operate brick-and-mortar stores and online stores as well to sell various products. They introduce the ease and efficiency of shopping online by delivering products, often at lower prices, to the shoppers' front doors. Stefan et al. (2017) reported that one in five enterprises in the European Union made electronic sales in 2015. In the United States, online sales stood at \$263.3 billion and accounted for 5.6 percent of total retail sales in 2013 (Gorodnichenko et al., 2015).

Market segments are usually interdependent. Gensch and Welam (1974) noted that "there is interaction in terms of members of one segment observing messages intended for members of other segments, and in terms of direct communication among members of other segments." Hsiao (2009) explored how consumers evaluate the value of time, when they are facing a shopping mode choice between offline shopping and online shopping. It was found that "the value of delivery time for a purchased book from an online bookstore to a consumer is approximately $\$ 0.53$ per day, which means an online bookstore will have to lower a book's price by $\$ 0.53$ to attract a physical bookstore shopper if the delivery is delayed for one day." Some distinctive features of the offline and online segments are noted in the literature. For example, Gorodnichenko et al. (2015) found that online prices are more flexible than prices charged in offline stores. Fassnacht and Unterhuber (2016) provided experimental evidence that price differentiation with lower online prices is feasible. Because of the differences in the offline and online segments and their interactions, a profit-maximizing retailer needs to resolve the strategic issue of developing and implementing an optimal pricing strategy to sell a product in its offline and online stores. 
The practice of price differentiation is closely related to the concept of yield management that aims to maximize sales revenue by selling a product to the right customer at the optimal price. Two notable studies are relevant to our paper in the context of yield management. Zeng (2013) developed multiple pricing strategies for a typical retailer that sells a product in a market composed of experienced and inexperienced consumers. Lee and $\mathrm{Ng}$ (2001) analytically determined the optimal allocation of service capacity over a two-period planning horizon and corresponding pricing strategies for a monopolistic service provider. Our paper is in the spirit of Zeng (2013) and Lee \& Ng (2001), but different in three significant ways. First, in our paper the market served by a monopolistic retailer is composed of the offline and online segments. Two linear multivariate demand functions are employed to model the offline and online demands, respectively. Second, the necessary and sufficient conditions are provided for the retailer to set the optimal offline and online selling prices. Third, the impacts of the uniform delivery lead time on the retailer's optimal pricing scheme and profitability are analytically explored and then demonstrated in a numerical study with three experimental scenarios.

In the next section, we model the aggregate demand of each segment in the market and then formulate the sales revenue function. The optimal offline and online selling prices are analytically determined in the third section. The fourth section presents the computational results of the numerical study. Finally, the paper concludes with a summary of its findings, managerial implications, limitations, and directions for future research in the fifth section.

\section{MODEL FRAMEWORK}

Consider a monopolistic retailer that must determine the prices of an identical product to be sold in its brick-and-mortar and online stores, respectively. The market served by the retailer is divided into two segments: consumers who purchase the product in its brick-and-mortar stores (i.e., the offline segment) and those who make their purchases in its online stores (i.e., the online segment). We assume that in the offline segment, the units of the product purchased by a customer are immediately delivered at the time of the purchase; in the online segment, however, the delivery occurs within a uniform time frame guaranteed by the retailer after a customer makes a purchase.

The problems that we intend to solve in this paper can be specifically stated as follows: (i) What is the best pricing strategy of a retailer in a monopolistic environment to price an identical product to be sold in the offline and online segments during a selling season so that its total sales revenue will be maximized? (ii) What are the impacts of the uniform delivery lead time for online shopping on the retailer's optimal offline and online selling prices? (iii) What is the impact of the uniform delivery lead time for online shopping on the retailer's profitability?

We make the following basic assumptions while addressing the three strategic issues stated above: (i) All consumers in the two-segment market are well aware of the offline and online selling prices and the uniform delivery lead time for online shopping. (ii) The aggregate demand of each segment is affected by the offline and online selling prices and the delivery lead time as well. (iii) The retailer can fully satisfy the aggregate demand of each segment.

To improve exposition, the offline segment is denoted as Segment 1 and the online segment as Segment 2, respectively. Several terms used to model the demand of each segment and formulate the sales revenue function are defined below:

$R \quad$ the retailer's total sales revenue generated from the entire market;

$P_{i} \quad$ the price per unit of the product charged to Segment $i$ by the retailer $(i=1,2)$;

$d_{i} \quad$ the aggregate demand of Segment $i(i=1,2)$;

$L \quad$ the uniform delivery lead time for online shopping guaranteed by the retailer.

A linear form of the demand function is extensively employed in both theoretical and empirical studies (see Huang et al., 2013, for a review). Zufryden (1975) stressed that linear formulations are more appealing because of the relative ease of parameter estimation through classical statistical methods. As it is assumed that the aggregate demand of each segment is affected by the offline and online selling prices 
and the delivery lead time, we thus employ two linear multivariate demand functions based on the work of Huang et al. (2013) to model the aggregate demand $d_{i}(i=1,2)$ in our study:

$d_{1}=\alpha_{1}-\beta_{1} P_{1}+\beta_{12} P_{2}+\gamma_{1} L$

$d_{2}=\alpha_{2}-\beta_{2} P_{2}+\beta_{21} P_{1}-\gamma_{2} L$

where, $\alpha_{1}, \alpha_{2}, \beta_{1}, \beta_{2}, \beta_{12}, \beta_{21}, \gamma_{1}, \gamma_{2}>0$.

For $i=1,2$, the constant $\alpha_{i}$ captures the part of the aggregate demand of Segment $i$ that does not vary with the offline and online prices (i.e., $P_{1}$ and $P_{2}$ ) and the delivery lead time (i.e., $L$ ); $\beta_{i}$ measures the price sensitivity of demand of Segment $i$ to changes in the price charged to the same segment, $P_{i}$. $\beta_{12}$ measures the price sensitivity of demand of Segment 1 to changes in the price charged to Segment $2, P_{2}$. Similarly, $\beta_{21}$ measures the price sensitivity of demand of Segment 2 to changes in $P_{l} \cdot \gamma_{\mathrm{i}}$ is the delivery lead time sensitivity of demand of Segment $i$.

Expression (1) shows that the aggregate demand of Segment $1, d_{1}$, is decreasing in $P_{1}$ but increasing in $P_{2}$. In contrast, the aggregate demand of Segment $2, d_{2}$, is decreasing in $P_{2}$ but increasing in $P_{1}$, as noted in expression (2). These functional relationships could be found in the offline and online sales of certain products. For example, everything else being constant, more students would purchase a required textbook online if its offline price goes up. On the other hand, a higher online price may boost the offline demand for the textbook.

As noted in expression (1), the aggregate demand of Segment $1, d_{l}$, is increasing in $L$. In contrast, expression (2) shows that the aggregate demand of Segment $2, d_{2}$, is decreasing in $L$. This phenomenon is often observed in the offline and online market segments for some products: as the delivery lead time for online shopping gets longer, more consumers would shop in brick-and-mortar stores rather than online.

Based on the assumption that the aggregate demand of each segment can be fully satisfied by the retailer, the total sales revenue generated from the two-segment market is given by expression (3):

$R=P_{1} d_{1}+P_{2} d_{2}$

Substituting expressions (1) and (2) in (3) yields:

$R=\left(\alpha_{1}-\beta_{1} P_{1}+\beta_{12} P_{2}+\gamma_{1} L\right) P_{1}+\left(\alpha_{2}-\beta_{2} P_{2}+\beta_{21} P_{1}-\gamma_{2} L\right) P_{2}$

Expression (4) shows that the retailer's total sales revenue, $R$, is a multivariate quadratic function of the offline and online selling prices, $P_{1}$ and $P_{2}$, and the uniform delivery lead time, $L$. Based on (4), we employ an approach of differential calculus to find out the optimal offline and online selling prices, $P_{1}{ }^{*}$ and $P_{2}{ }_{2}$, at which the retailer's total sales revenue is maximized.

\section{OPTIMAL OFFLINE AND ONLINE SELLING PRICES}

In this section, we first determine the optimal offline and online selling prices in closed forms, and subsequently examine the impacts of the uniform delivery lead time on the retailer's optimal selling prices and total sales revenue. As a result, four propositions are introduced below for which the proofs are found in the Appendix.

Proposition 1. Given $4 \beta_{1} \beta_{2}-\left(\beta_{12}+\beta_{21}\right)^{2}>0, \alpha_{2}\left(\beta_{12}+\beta_{21}\right)+2 \alpha_{1} \beta_{2}+\left[2 \beta_{2} \gamma_{1}-\left(\beta_{12}+\beta_{21}\right) \gamma_{2}\right] L>0$, and $\alpha_{1}\left(\beta_{12}+\beta_{21}\right)+2 \alpha_{2} \beta_{1}-\left[2 \beta_{1} \gamma_{2}-\left(\beta_{12}+\beta_{21}\right) \gamma_{1}\right] L>0$, the retailer's total sales revenue, $R$, reaches its maximal level at

$P_{1}^{*}=\frac{\alpha_{2}\left(\beta_{12}+\beta_{21}\right)+2 \alpha_{1} \beta_{2}+\left[2 \beta_{2} \gamma_{1}-\left(\beta_{12}+\beta_{21}\right) \gamma_{2}\right] L}{4 \beta_{1} \beta_{2}-\left(\beta_{12}+\beta_{21}\right)^{2}}>0$, 
and

$$
P_{2}^{*}=\frac{\alpha_{1}\left(\beta_{12}+\beta_{21}\right)+2 \alpha_{2} \beta_{1}-\left[2 \beta_{1} \gamma_{2}-\left(\beta_{12}+\beta_{21}\right) \gamma_{1}\right] L}{4 \beta_{1} \beta_{2}-\left(\beta_{12}+\beta_{21}\right)^{2}}>0
$$

Proposition 2. Given that $4 \beta_{1} \beta_{2}-\left(\beta_{12}+\beta_{21}\right)^{2}>0$ and all other things being equal:
a. If $\frac{\gamma_{1}}{\gamma_{2}}>\frac{\beta_{12}+\beta_{21}}{2 \beta_{2}}$, then $P_{1}{ }^{*}$ is monotonically increasing in $L$.
b. If $\frac{\gamma_{1}}{\gamma_{2}}<\frac{\beta_{12}+\beta_{21}}{2 \beta_{2}}$, then $P_{1}{ }^{*}$ is monotonically decreasing in $L$.
c. If $\frac{\gamma_{1}}{\gamma_{2}}=\frac{\beta_{12}+\beta_{21}}{2 \beta_{2}}$, then $P_{1}^{*}$ is constant in $L$.

Proposition 3. Given that $4 \beta_{1} \beta_{2}-\left(\beta_{12}+\beta_{21}\right)^{2}>0$ and all other things being equal:
a. If $\frac{\gamma_{2}}{\gamma_{1}}>\frac{\beta_{12}+\beta_{21}}{2 \beta_{1}}$, then $P_{2}{ }^{*}$ is monotonically decreasing in $L$.
b. If $\frac{\gamma_{2}}{\gamma_{1}}<\frac{\beta_{12}+\beta_{21}}{2 \beta_{1}}$, then $P_{2}^{*}$ is monotonically increasing in $L$.
c. If $\frac{\gamma_{2}}{\gamma_{1}}=\frac{\beta_{12}+\beta_{21}}{2 \beta_{1}}$, then $P_{2}{ }^{*}$ is constant in $L$.

Proposition 4. Given that the retailer's total sales revenue, $R$, is determined by expression (4):
a. If $\frac{\gamma_{1}}{\gamma_{2}}>\frac{P_{2}}{P_{1}}$, then $R$ is monotonically increasing in $L$.
b. If $\frac{\gamma_{1}}{\gamma_{2}}<\frac{P_{2}}{P_{1}}$, then $R$ is monotonically decreasing in $L$.
c. If $\frac{\gamma_{1}}{\gamma_{2}}=\frac{P_{2}}{P_{1}}$, then $R$ is constant in $L$.

Proposition 1 provides the necessary and sufficient conditions for the retailer to optimize its total sales revenue in the two-segment market. It is noted in expressions (5) and (6) that the optimal offline and online selling prices, $P_{1}{ }^{*}$ and $P_{2}{ }^{*}$, are determined by the price sensitivities of each segment, the two constants, $\alpha_{1}$ and $\alpha_{2}$, and the delivery lead time, $L$. With the optimal selling prices $P_{1}{ }^{*}$ and $P_{2}{ }^{*}$ being established, the retailer can determine the demand of each segment, $d_{i}^{*}(i=1,2)$ by substituting $P_{1}{ }^{*}$ and $P_{2}{ }^{*}$ in expressions (1) and (2), respectively. If the retailer sells the product exactly at the levels of $d_{1}{ }^{*}$ and $d_{2}{ }^{*}$, the total sales revenue, $R$, will reach the optimal level, $R^{*}=P_{1}{ }^{*} d_{1}{ }^{*}+P_{2}{ }^{*} d_{2}{ }^{*}$.

Propositions 2 and 3 offer guidelines for the retailer to fine-tune the offline and online selling prices as the delivery lead time changes. Proposition 4 gives the direction of adjusting the delivery lead time for the retailer to enhance its profitability.

\section{NUMERICAL ILLUSTRATIONS}

A numerical study is presented in this section to examine the impacts of the uniform delivery lead time for online shopping on the retailer's optimal offline and online prices and total sales revenue. For illustrative purposes, the values of the nine model parameters are selected as follows:

$$
\begin{aligned}
& \alpha_{1}=2,000 \text { units, } \alpha_{2}=2,000 \text { units, } \beta_{1}=3.5, \beta_{2}=3.5, \beta_{12}=3.0, \beta_{21}=3.0, \\
& \gamma_{1}=200,250, \\
& \gamma_{2}=200,250, \\
& L=0,1,2,3,4,5,6,7 \text { days. }
\end{aligned}
$$


In the numerical study, the optimal offline and online prices, $P_{1}{ }^{*}$ and $P_{2}{ }^{*}$, are calculated based on expressions (5) and (6). The optimal demand levels in Segments 1 and $2, d_{1}{ }^{*}$ and $d_{2}{ }^{*}$, are determined by substituting $P_{1}{ }^{*}$ and $P_{2}{ }^{*}$ into expressions (1) and (2). Then, substituting $P_{1}{ }^{*}, P_{2}{ }^{*}, d_{1}{ }^{*}$, and $d_{2}{ }^{*}$ into expression (3) yields the optimal total sales revenue, $R^{*}$. The computational results for three experimental scenarios are reported in Tables 1, 2, and 3, respectively.

As shown in Table 1, for example, the optimal pricing scheme $\left(P_{1}{ }^{*}, P_{2}{ }^{*}\right)=(\$ 2107.69, \$ 1892.31)$ is calculated for $\gamma_{1}=200$ and $\gamma_{2}=200$ in conjunction with the delivery lead time $L=7$ days, and consequently, the optimal total sales revenue, $R^{*}$, is $\$ 4,150,769$. Table 1 shows that as the delivery lead time, $L$, increases, $P_{1}{ }^{*}$ goes up while $P_{2}{ }^{*}$ goes down, increasing $R^{*}$. Table 2 shows that as $L$ increases, both $P_{1}{ }^{*}$ and $P_{2}{ }^{*}$ go up, also increasing $R^{*}$. In contrast, Table 3 shows that as $L$ increases, both $P_{1}{ }^{*}$ and $P_{2}{ }^{*}$ go down, lowering $R^{*}$. These simulation results are consistent with Propositions 2, 3, and 4.

TABLE 1

OPTIMAL PRICES, PRICE DIFFERENCE AND TOTAL SALES REVENUE FOR $\gamma_{1}=200$ AND $\gamma_{2}=200$

\begin{tabular}{|c|c|c|c|c|}
\hline \multirow{2}{*}{$\begin{array}{c}\text { Delivery } \\
\text { lead time, } L \\
\text { (days) }\end{array}$} & \multicolumn{2}{|c|}{$\begin{array}{c}\text { Optimal prices } \\
\text { (\$/unit) }\end{array}$} & $\begin{array}{c}\text { Price difference } \\
\text { (\$/unit) }\end{array}$ & $\begin{array}{c}\text { Total sales } \\
\text { revenue }(\$)\end{array}$ \\
\cline { 2 - 5 } & $P_{1}{ }^{*}$ & $P_{2}{ }^{*}$ & $P_{1}{ }^{*}-P_{2}{ }^{*}$ & $R^{*}$ \\
\hline 0 & 2000.00 & 2000.00 & 0.00 & 4000000 \\
\hline 1 & 2015.38 & 1984.62 & 30.76 & 4003077 \\
\hline 2 & 2030.77 & 1969.23 & 61.54 & 4012308 \\
\hline 3 & 2046.15 & 1953.85 & 92.30 & 4027692 \\
\hline 4 & 2061.54 & 1938.46 & 123.08 & 4049231 \\
\hline 5 & 2076.92 & 1923.08 & 153.84 & 4076923 \\
\hline 6 & 2092.31 & 1907.69 & 184.62 & 4110769 \\
\hline 7 & 2107.69 & 1892.31 & 215.38 & 4150769 \\
\hline
\end{tabular}

TABLE 2

OPTIMAL PRICES, PRICE DIFFERENCE AND TOTAL SALES REVENUE FOR $\gamma_{1}=250$ AND $\gamma_{2}=200$

\begin{tabular}{|c|c|c|c|c|}
\hline \multirow{2}{*}{$\begin{array}{c}\text { Delivery } \\
\text { eead time, } L \\
\text { (days) }\end{array}$} & \multicolumn{2}{|c|}{$\begin{array}{c}\text { Optimal prices } \\
\text { (\$/unit) }\end{array}$} & $\begin{array}{c}\text { Price difference } \\
\text { (\$/unit) }\end{array}$ & $\begin{array}{c}\text { Total sales } \\
\text { revenue }(\$)\end{array}$ \\
\cline { 2 - 5 } & $P_{1}{ }^{*}$ & $P_{2}{ }^{*}$ & $P_{1}{ }^{*}-P_{2}{ }^{*}$ & $R^{*}$ \\
\hline 0 & 2000.00 & 2000.00 & 0.00 & 4000000 \\
\hline 1 & 2042.31 & 2007.69 & 34.62 & 4104519 \\
\hline 2 & 2084.62 & 2015.38 & 69.24 & 4218077 \\
\hline 3 & 2126.92 & 2023.08 & 103.84 & 4340673 \\
\hline 4 & 2169.23 & 2030.77 & 138.46 & 4472308 \\
\hline 5 & 2211.54 & 2038.46 & 173.08 & 4612981 \\
\hline 6 & 2253.85 & 2046.15 & 207.70 & 4762692 \\
\hline 7 & 2296.15 & 2053.85 & 242.30 & 4921442 \\
\hline
\end{tabular}


TABLE 3

OPTIMAL PRICES, PRICE DIFFERENCE AND TOTAL SALES REVENUE FOR $\gamma_{1}=200$ AND $\gamma_{2}=250$

\begin{tabular}{|c|c|c|c|c|}
\hline \multirow{2}{*}{$\begin{array}{c}\text { Delivery } \\
\text { lead time, } L \\
\text { (days) }\end{array}$} & \multicolumn{2}{|c|}{$\begin{array}{c}\text { Optimal prices } \\
\text { (\$/unit) }\end{array}$} & $\begin{array}{c}\text { Price difference } \\
\text { (\$/unit) }\end{array}$ & $\begin{array}{c}\text { Total sales } \\
\text { revenue }(\$)\end{array}$ \\
\cline { 2 - 5 } & $P_{1}{ }^{*}$ & $P_{2}{ }^{*}$ & $P_{1}{ }^{*}-P_{2}{ }^{*}$ & $R^{*}$ \\
\hline 0 & 2000.00 & 2000.00 & 0.00 & 4000000 \\
\hline 1 & 1992.31 & 1957.69 & 34.62 & 3904519 \\
\hline 2 & 1984.62 & 1915.38 & 69.24 & 3818077 \\
\hline 3 & 1976.92 & 1873.08 & 103.84 & 3740673 \\
\hline 4 & 1969.23 & 1830.77 & 138.46 & 3672308 \\
\hline 5 & 1961.54 & 1788.46 & 173.08 & 3612981 \\
\hline 6 & 1953.85 & 1746.15 & 207.70 & 3562692 \\
\hline 7 & 1946.15 & 1703.85 & 242.30 & 3521442 \\
\hline
\end{tabular}

\section{CONCLUSIONS}

In this paper, two linear deterministic demand functions are employed to describe the relationships of the offline and online demands with the selling prices and the uniform delivery lead time for online shopping, respectively. We first address the problem of determining the optimal offline and online selling prices for a monopolistic retailer to maximize its total sales revenue. The impacts of the delivery lead time on the retailer's optimal pricing scheme and total sales revenue are analytically explored and then demonstrated in a numerical study.

The analytical findings presented in the third section imply that the retailer should take the delivery lead time and all the four types of price sensitivity into consideration when making its pricing decisions. In particular, as the delivery lead time changes, both the offline and online selling prices should be adjusted in the right directions.

There are several directions for future research. First, the demand functions employed in our model framework have a deterministic structure. Probabilistic demand functions may be chosen for model development. Second, the offline and online demands could be modeled with non-linear multivariate functions. Third, in this exploratory study we focus on a retailer in a monopolistic environment. Incorporating competition into the model framework would be a possible extension. 


\section{REFERENCES}

Fassnacht, M., \& Unterhuber, S. (2016). Consumer response to online/offline price differentiation. Journal of retailing \& Consumer Services, 28, 137-148.

Gensch, D. H., \& Welam, U. P. (1974). Optimal price and promotion for interdependent market segments. Operations Research, 22(4), 746-755.

Gorodnichenko, Y., Sheremirov, V., \& Talavera, O. (2015). Price setting in online markets: Does it click? Working Paper Series (Federal Reserve Bank of Boston), 15(1), 1-60.

Hsiao, M. H. (2009). Shopping mode choice: Physical store shopping versus e-shopping. Transportation Research Part E, 45, 86-95.

Huang, J., Leng, M., \& Parlar, M. (2013). Demand functions in decision modeling: A comprehensive survey and research directions. Decision Sciences, 44(3), 557-609.

Lee, K. S., \& Ng, I. C. L. (2001). Advance sale of service capacities: Theoretical analysis of the impact of price sensitivity on pricing and capacity allocations. Journal of Business Research, 54, 219-225.

Stefan, M. C., Andreiana V. A., \& Panagoret, I. (2017). The role of social networks in the evolution of online sales - study case. Journal of Science and Arts, 4(41), 731-744.

Zeng, C. (2013). Optimal advance selling strategy under price commitment. Pacific Economic Review, $18(2), 233-258$.

Zufryden, F. S. (1975). Optimal multi-period advertising budget allocation within a competitive environment. Operational Research Quarterly, 26(4), 743-754. 


\section{APPENDIX}

\section{Proof of Proposition 1}

The first-order necessary condition for optimality implies that

$\frac{\partial R}{\partial P_{1}}=\alpha_{1}-2 \beta_{1} P_{1}+\left(\beta_{12}+\beta_{21}\right) P_{2}+\gamma_{1} L=0 ;$

$\frac{\partial R}{\partial P_{2}}=\alpha_{2}-2 \beta_{2} P_{2}+\left(\beta_{12}+\beta_{21}\right) P_{1}-\gamma_{2} L=0$.

Solving equations (A.1) and (A.2) simultaneously, we obtain

$P_{1}^{*}=\frac{\alpha_{2}\left(\beta_{12}+\beta_{21}\right)+2 \alpha_{1} \beta_{2}+\left[2 \beta_{2} \gamma_{1}-\left(\beta_{12}+\beta_{21}\right) \gamma_{2}\right] L}{4 \beta_{1} \beta_{2}-\left(\beta_{12}+\beta_{21}\right)^{2}}$,

and

$P_{2}^{*}=\frac{\alpha_{1}\left(\beta_{12}+\beta_{21}\right)+2 \alpha_{2} \beta_{1}-\left[2 \beta_{1} \gamma_{2}-\left(\beta_{12}+\beta_{21}\right) \gamma_{1}\right] L}{4 \beta_{1} \beta_{2}-\left(\beta_{12}+\beta_{21}\right)^{2}}$.

Since $\partial^{2} R / \partial P_{1}{ }^{2}=-2 \beta_{1}<0, \partial^{2} R / \partial P_{2}{ }^{2}=-2 \beta_{2}<0, \partial^{2} R / \partial P_{1} \partial P_{2}=\beta_{12}+\beta_{21}$, and $\partial^{2} R / \partial P_{2} \partial P_{1}=\beta_{12}+\beta_{21}$, $\left(\partial^{2} R / \partial P_{1}^{2}\right)\left(\partial^{2} R / \partial P_{2}^{2}\right)-\left(\partial^{2} R / \partial P_{1} \partial P_{2}\right)\left(\partial^{2} R / \partial P_{2} \partial P_{1}\right)=4 \beta_{1} \beta_{2}-\left(\beta_{12}+\beta_{21}\right)^{2}>0$. This indicates that the total sales revenue, $R$, reaches its maximal level at $P_{1}{ }^{*}$ and $P_{2}{ }^{*}$. In addition, the condition that $4 \beta_{1} \beta_{2}-\left(\beta_{12}+\right.$ $\left.\beta_{21}\right)^{2}>0, \alpha_{2}\left(\beta_{12}+\beta_{21}\right)+2 \alpha_{1} \beta_{2}+\left[2 \beta_{2} \gamma_{1}-\left(\beta_{12}+\beta_{21}\right) \gamma_{2}\right] L>0$, and $\alpha_{1}\left(\beta_{12}+\beta_{21}\right)+2 \alpha_{2} \beta_{1}-\left[2 \beta_{1} \gamma_{2}-\left(\beta_{12}+\right.\right.$ $\left.\left.\beta_{21}\right) \gamma_{1}\right] L>0$, implies $P_{1}^{*}>0$ and $P_{2}^{*}>0$.

\section{Proof of Proposition 2}

From expression (5), we obtain the first partial derivative with respect to $L$ :

$\frac{\partial P_{1}^{*}}{\partial L}=\frac{2 \beta_{2} \gamma_{1}-\left(\beta_{12}+\beta_{21}\right) \gamma_{2}}{4 \beta_{1} \beta_{2}-\left(\beta_{12}+\beta_{21}\right)^{2}}$.

Given $4 \beta_{1} \beta_{2}-\left(\beta_{12}+\beta_{21}\right)^{2}>0$ :
a. If $\frac{\gamma_{1}}{\gamma_{2}}>\frac{\beta_{12}+\beta_{21}}{2 \beta_{2}}$, then $\partial P_{1}{ }^{*} / \partial L>0$ and hence, $P_{1}{ }^{*}$ is monotonically increasing in $L$.
b. If $\frac{\gamma_{1}}{\gamma_{2}}<\frac{\beta_{12}+\beta_{21}}{2 \beta_{2}}$, then $\partial P_{1}{ }^{*} / \partial L<0$ and hence, $P_{1}{ }^{*}$ is monotonically decreasing in $L$.
c. If $\frac{\gamma_{1}}{\gamma_{2}}=\frac{\beta_{12}+\beta_{21}}{2 \beta_{2}}$, then $\partial P_{1}{ }^{*} / \partial L=0$ and hence, $P_{1}{ }^{*}$ is constant in $L$.

\section{Proof of Proposition 3}

From expression (6), we obtain the first partial derivative with respect to $L$ :

$\frac{\partial P_{2}{ }^{*}}{\partial L}=\frac{-2 \beta_{1} \gamma_{2}+\left(\beta_{12}+\beta_{21}\right) \gamma_{1}}{4 \beta_{1} \beta_{2}-\left(\beta_{12}+\beta_{21}\right)^{2}}$.

Given $4 \beta_{1} \beta_{2}-\left(\beta_{12}+\beta_{21}\right)^{2}>0$ :
a. If $\frac{\gamma_{2}}{\gamma_{1}}>\frac{\beta_{12}+\beta_{21}}{2 \beta_{1}}$, then $\partial P_{2}{ }^{*} / \partial L<0$ and hence, $P_{2}{ }^{*}$ is monotonically decreasing in $L$.
b. If $\frac{\gamma_{2}}{\gamma_{1}}<\frac{\beta_{12}+\beta_{21}}{2 \beta_{1}}$, then $\partial P_{2}{ }^{*} / \partial L>0$ and hence, $P_{2}{ }^{*}$ is monotonically increasing in $L$. 
c. If $\frac{\gamma_{2}}{\gamma_{1}}=\frac{\beta_{12}+\beta_{21}}{2 \beta_{1}}$, then $\partial P_{2}{ }^{*} / \partial L=0$ and hence, $P_{2}{ }^{*}$ is constant in $L$.

\section{Proof of Proposition 4}

From expression (4), we obtain the first-order partial derivative with respect to $L$ :

$\frac{\partial R}{\partial L}=\gamma_{1} P_{1}-\gamma_{2} P_{2}$
a. If $\frac{\gamma_{1}}{\gamma_{2}}>\frac{P_{2}}{P_{1}}$, then $\partial R / \partial L>0$ and hence, $R$ is monotonically increasing in $L$.
b. If $\frac{\gamma_{1}}{\gamma_{2}}<\frac{P_{2}}{P_{1}}$, then $\partial R / \partial L<0$ and hence, $R$ is monotonically decreasing in $L$.
c. If $\frac{\gamma_{1}}{\gamma_{2}}=\frac{P_{2}}{P_{1}}$, then $\partial R / \partial L=0$, and hence, $R$ is constant in $L$. 